\title{
BMJ Open Randomised factorial mixed method pilot study of aerobic and resistance exercise in haemodialysis patients: DIALY-SIZE!
}

\author{
Stephanie Thompson, ${ }^{1}$ Scott Klarenbach, ${ }^{1}$ Anita Molzahn, ${ }^{2}$ Anita Lloyd, ${ }^{3}$ \\ Iwona Gabrys, ${ }^{4}$ Mark Haykowsky, ${ }^{5}$ Marcello Tonelli ${ }^{6}$
}

To cite: Thompson S, Klarenbach S, Molzahn A, et al. Randomised factorial mixed method pilot study of aerobic and resistance exercise in haemodialysis patients: DIALY-SIZE!. BMJ Open 2016;6:e012085. doi:10.1136/bmjopen-2016012085

- Prepublication history for this paper is available online. To view these files please visit the journal online (http://dx.doi.org/10.1136/ bmjopen-2016-012085).

Received 29 March 2016 Revised 13 June 2016 Accepted 5 August 2016

For numbered affiliations see end of article.

Correspondence to Dr Stephanie Thompson; th11@ualberta.ca

\section{ABSTRACT}

Objectives: For people with end-stage renal disease requiring haemodialysis, exercise can improve aspects of quality of life (QoL). However, the relative benefits and risks of different types of exercise in this population are unknown. Therefore, this pilot study aimed to evaluate the feasibility of a main study evaluating the efficacy of cycling and resistance exercise each performed during the haemodialysis treatment on QoL.

Methods: In this factorial (2×2) pilot trial, 31 haemodialysis patients were randomised to cycling, resistance, cycling and resistance, or an attention control. Feasibility was defined a priori by criteria on recruitment, fidelity to the protocol and patient response to the intervention. To better understand feasibility, we conducted interviews with dialysis unit staff and trial participants. As secondary outcomes, we estimated the main effect of cycling and weights each compared with control on QoL, physical function and strength.

Findings: We exceeded the target accrual of 28 participants over 12 weeks. Irrespective of exercise group allocation, adherence was high; of the 1038 training sessions offered, $87 \%$ were initiated and over $80 \%$ of exercise sessions were performed as per protocol. Progression based on perceived exertion, individual instruction and interactions with the kinesiologist facilitated acceptability across exercise groups. Using an attention control, measures of contamination and attrition were low. Important barriers to unit staff readiness for the intervention were initial safety and workflow concerns, unit workload and onerous data collection. Secondary outcomes were not statistically significant. Adverse events were low and did not increase with a higher volume of exercise.

Conclusions: The main study is feasible with minor modifications. In addition to practical assistance, involvement from unit staff could increase patient participation and improve trial implementation. Strategies to increase acceptability of the intervention for staff include improving workflow integration and using a prestudy demonstration phase to introduce the intervention.

Trial registration number: NCT02234232. Results

\section{Strengths and limitations of this study}

- To our knowledge, this work is the first to include qualitative methods in evaluating the design of an exercise trial in haemodialysis patients.

- Understanding patient and dialysis unit staff perspectives on trial processes and the intervention was critical in identifying barriers to trial implementation and for purposing strategies to improve these.

- Detailed information on aspects of trial delivery contributes useful knowledge to the renal exercise literature on how key methodological and practical limitations could be feasibly improved in order to increase trial quality, relevance and potentially effectiveness.

- Owing to limited sample size, we could not triangulate patients' experiences of intervention factors by high or low adherence.

- Our focus was on identifying the factors that influenced trial implementation and how these factors would influence the longer-term sustainability of the intervention is not clear.

\section{INTRODUCTION}

While haemodialysis (HD) is a life-sustaining therapy for people with end-stage renal disease (ESRD), it is associated with low quality of life (QoL) ${ }^{12}$ and a marked decline in functional status. ${ }^{3}$ Although the benefits of exercise in this population have been recognised, few studies have evaluated how different types of exercise can influence QoL, and the majority of interventions have evaluated aerobic exercise. ${ }^{4}{ }^{5}$ How to most effectively engage patients in the optimal exercise prescription and achieve the desired outcome while minimising risk, is critical to increasing patient participation.

Many generic QoL scales used in exercise studies in people with ESRD address the individual's perception of their ability to meet the demands of everyday living. However, 
performance of daily tasks is more dependent on musculoskeletal fitness than aerobic capacity. ${ }^{6}{ }^{7}$ In the elderly non-ESRD population ${ }^{68}$ and in people with congestive heart failure $(\mathrm{CHF}),{ }^{9}$ resistance training is a promising means of improving QoL and decreasing disability. However, whether resistance training confers specific benefits relevant to aspects of QoL in people with ESRD is not known.

The aim of our future multicentre study is to evaluate the effect of two types of exercise (cycling and resistance) each compared with control and performed during the HD treatment (intradialytic exercise, (IDE)) on QoL and physical performance using a randomised factorial design. Prior to proceeding with this main study, a pilot was warranted to evaluate the feasibility of the design. Although delivering exercise during HD has been associated with greater adherence compared with a home-based exercise programme ${ }^{10}$ few pilot studies have rigorously evaluated the feasibility or the integrity of trial implementation and we are not aware of any studies that have included qualitative methods to provide a more comprehensive understanding of the implementation process. ${ }^{11}$

\section{METHODS}

Study design

This mixed methods, single-centre, randomised, factorial $(2 \times 2)$ trial included qualitative interviews with trial participants and dialysis unit staff to evaluate domains of feasibility defined a priori: recruitment, fidelity to the study protocol and the response of trial participants and dialysis unit staff to the intervention. In a secondary analysis, we explored differences in QoL, physical function and strength. The two factors evaluated were aerobic exercise (cycling) and resistance exercise (leg weights). HD patients were randomised to one of four groups: cycling, leg weights, combined leg weights and cycling, or stretching (an attention control). The rationale for using a factorial design is for the efficiency of testing more than one intervention in the same participants. ${ }^{12}$ (There is no known interaction between aerobic and resistance exercise in the literature ie, the effect of aerobic exercise does not differ in the presence of resistance exercise.) All exercises were performed during HD at each thrice-weekly dialysis session over 12 weeks (36 sessions). The study protocol was registered under NCT02234232.

\section{Setting and participants}

The trial setting was an outpatient dialysis unit in Edmonton, Canada that serves $\sim 110$ patients. A study coordinator recruited participants during their HD sessions. Inclusion criteria were as follows: adult (age $\geq 18$ ); dialysis dependent for $\geq 3$ consecutive months; receiving $\geq 3$ dialysis treatments per week; mobile (any distance, walking aid permitted); at least one non-prosthetic limb; and capable of providing consent. Exclusion criteria were as follows: currently enrolled in a clinical trial; missing an average of $>2$ dialysis sessions per month; planned move or modality change within the next 4 months; currently enrolled in a structured exercise programme; scheduled hospitalisation for >1 week; unstable during $\mathrm{HD}$; and any uncontrolled medical condition that would preclude participation in a low/ moderate intensity exercise programme. ${ }^{13}$

\section{Randomisation and blinding}

Participants were randomised on a 1:1:1:1 ratio using a computerised randomisation procedure with permuted blocks of eight and twelve. Allocation was concealed in serially numbered, opaque, sealed envelopes. The randomisation list was generated by the statistician and kept in a locked cabinet. Given the open setting of the dialysis unit and the nature of the intervention, it was not logistically possible to blind the participants or the kinesiologist to treatment allocation. Therefore, participants and HD unit staff were blinded to the study hypothesis. Patients were informed that they would be randomised to one of four different exercise regimens; a stretching exercise group served as the attention control. Kinesiologists assessed all tests of physical performance; a blinded assessor performed outcome assessments at 12 weeks.

\section{Exercise intervention}

A kinesiologist instructed all participants on how to perform exercises and supervised a minimum of two of the participants' thrice-weekly exercise sessions. In addition, the kinesiologist supervised the first three exercise sessions and the first session following progression of the exercise prescription. When the kinesiologist was not present, dialysis unit staff assisted patients with equipment set-up and completed trial documentation. Throughout the study, unit staff were also asked to help motivate patients by providing verbal encouragement. The kinesiologist instructed all participants on how to use rating of perceived exertion (RPE) with the Borg scale (6-20). ${ }^{14}$ The intensity of exercise for the aerobic, resistance and combined intervention groups was prescribed at a level of 12-14 or 'somewhat hard' on the Borg (RPE) scale and a RPE level of 8-9 ('very light') for the stretching group.

\section{Aerobic intervention}

Each session included a 5 min warm-up and cool-down on the cycle ergometer at an RPE of 9-11. The cycling protocol started with $15 \mathrm{~min}$ of cycling with time increased by $2.5 \mathrm{~min}$ each week. The resistance was adjusted to maintain the target RPE. One of two types of cycle ergometers were used according to compatibility with the type of dialysis chair: the Monark $881 \mathrm{E}$ cycle (Healthcare International, Langley, Washington, USA) or the TherapyTrainer (Interactive Motivation, Greeley, Colorado, USA). 
Resistance intervention

Ankle weights (Fabrication Enterprises, White Plains, New York, USA) were used for knee extension, knee flexion and hip flexion. A Thera-Band (Hygenic Corporation, Akron, Ohio, USA) was used for hip abduction. Each session included a warm-up of one set of the four exercises against gravity. Based on RPE, exercises progressed from one set of 10-15 repetitions up to three sets. Weight or resistance was increased when the patient's RPE was less than target.

\section{Combined intervention}

Participants in the combined training group performed the full resistance exercise programme followed by the complete cycling programme.

\section{Attention control}

To equalise the effect of cointerventions, ${ }^{15}$ the control group performed a non-progressive stretching routine during dialysis. Participants performed two sets, each of four exercises as follows: pelvic tilts, gluteal stretch, calf and hamstring stretch. A Thera-Band Stretch Strap (Hygenic Corporation, Akron, ohio, USA) was used for the calf and hamstring stretches.

\section{Data collection}

Clinical data were collected at baseline via interviews with participants and chart review. Survey data, questionnaires and tests of physical performance were performed at baseline and at 12 weeks. At each session, the following data were recorded on exercise data collection forms (DCFs): pre-exercise and postexercise blood glucose (for diabetics), heart rate (HR), blood pressure (BP), reason for exercise non-participation and early termination, if applicable. During exercise, HR, BP and RPE were documented every $5 \mathrm{~min}$. Data on adverse events (AEs) were collected via interview at each exercise session with the kinesiologist and by chart review.

\section{Primary outcomes}

The primary outcome of feasibility was defined by a priori criteria (table 1) and focused on the following: recruitment (rate of accrual, reason for non-participation); fidelity to the protocol (dropout, adherence); response to the intervention (physical activity level outside of the dialysis unit, adoption of the other group's exercise (contamination)) and acceptability of the intervention.

\section{Recruitment}

Previous IDE trials report $20-46 \%$ of screened patients were randomised. ${ }^{16-19}$ We estimated that $\sim 85 \%$ of the 110 patients in this unit would be available for screening and targeted recruiting 28 participants. Based on the assumption that interested patients may already have preferences concerning exercise that would make randomisation undesirable, unwillingness to be randomised to exercise type was selected as a feasibility criterion.
Reason for non-participation in the trial was based on self-report.

\section{Fidelity to the protocol}

Based on dropout rates from exercise randomised control trials (RCTs) in people with chronic kidney disease, we defined a high dropout as $\geq 25 \%$ of the study population. ${ }^{4}$ Any participant who left the study at any time prior to completing the 12-week exercise programme was defined as a dropout. Adherence was measured to assess patients' willingness to participate in IDE and to ascertain if the exercises were performed as per protocol (table 1).

\section{Response to the intervention}

Acceptability of the exercises was defined as $\geq 50 \%$ of participants reporting that they would like to continue their current IDE programme after the trial is over. The change in physical activity performed outside of dialysis time was measured by self-reported questionnaire and using the Human Activity Profile (HAP) ${ }^{20}$ To evaluate whether any participants adopted the other group's intervention (contamination) outside of dialysis time, patients' completed questionnaires on the types of activities performed in their leisure time at baseline at 12 weeks.

\section{Qualitative interviews}

Detailed information on participants and data collection methods can be found elsewhere. ${ }^{21}$ To evaluate barriers to IDE implementation and to inform the content of staff in servicing, we interviewed dialysis unit staff 3 months prior to the start of the trial. To better understand the feasibility of unit staff participation in the delivery of the trial, unit staff members were also interviewed 4 months into the 6 -month trial. Unit staff were eligible to participate if the RCT directly affected their workflow and if they had worked in the unit during the trial. Interviews with RCT participants were conducted post-trial participation. All RCT participants were eligible if they were capable of sharing their experiences. Interviews were semistructured with open-ended questions followed by specific prompts on aspects of feasibility. All interviews were audiotaped and transcribed verbatim. For this analysis, interviews were coded using predetermined categories corresponding to our areas of feasibility and analysed to yield a descriptive summary of study findings.

\section{Secondary outcomes}

Secondary outcomes were as follows: QoL (the physical component summary (PCS) and the mental component summary (MCS)); tests of physical performance (short physical performance battery (SPPB), $30 \mathrm{~s}$ sit-to-stand test and 6 min walk test (6MWT)); an objective measure of strength; and AEs. Testing was carried out at baseline and at 12 weeks, pre-HD on their scheduled HD day. 
Table 1 A priori feasibility criteria and outcomes

Feasibility criteria
Recruitment
Accrual: 28 participants over 12 weeks
Reason for non-participation: proportion of screened
patients unwilling to be randomised must be $\leq 20 \%$
Fidelity to the protocol
Drop out: $\leq 25 \%$ of study participants withdrawing
participation

Adherence (willingness of participants to participate): of all exercise sessions offered, ${ }^{*} \geq 70 \%$ were initiated

Adherence (accordance with the exercise prescription): of all exercise sessions offered, $\geq 70 \%$ were performed at the prescribed time/volume and intensity

Impact of the intervention

Acceptability of the exercises: overall $\geq 50 \%$ of participants reporting that they would like to continue their current intradialytic exercise programme after the study is over

Change in the amount of physical activity performed overall: difference in the HAP scores between baseline and 12 weeks $\dagger$

Difference in the proportion of participants who reported never exercising outside of HD time

Contamination: any participant who adopted the exercise(s) of another intervention group during the study period

*Offered sessions exclude sessions lost to study dropout.

†Analysis performed for main effects adjusting for the baseline score and other factor.

AAS, adjusted activity score; HAP, Human Activity Profile; HD, haemodialysis; MAS, maximal activity score.
A total of 31 participants over 12 weeks

No patients reported randomisation to exercise type as a reason for non-participation.

A total of $16 \%$ of participants dropped out:

Cycling $n=1$, transplanted

Resistance $n=1$, injury from motor vehicle collision

Combined $n=1$, moved dialysis unit

Attention control $n=2$, nausea and vomiting; did not like exercise

A total of $87 \%$ of prescribed exercise sessions were initiated:

Cycling 89\%

Weights $83 \%$

Combined $90 \%$

Attention control 86\%

A total of $86 \%$ of prescribed exercise sessions were

performed as prescribed:

Cycling $87 \%$

Weights $84 \%$

Combined $88 \%$

Attention control $86 \%$

A total of $63 \%$ of participants said they would continue with their current exercise

Cycling 50\%

Weights $50 \%$

Combined $100 \%$

Stretching $38 \%$

MAS:

Cycling vs no cycling $4.3(-2.8,11.5) \mathrm{p}=0.3$

Weights vs no weights $-1.2(-8.4,6.0) p=0.7$

AAS:

Cycling vs no cycling $1.1(-7.7,9.9) \mathrm{p}=0.8$

Weights vs no weights $-0.9(-9.7,7.8) p=0.7$

Baseline: $39 \%$ of participants exercised almost never or never exercising vs 12 weeks: $29 \%$ of participants exercised almost never or never $(\mathrm{p}=0.55)$

No participants from the cycling, weights or stretching groups reported performing the other group's exercise

\section{Quality of life}

Participants completed The Kidney Disease Quality of Life Short Form 36 (KDQOL-SF 36). ${ }^{22}$ Item scores range from 0 to 100 , with higher scores being more favourable. For this pilot, only the mean difference in PCS and MCS are reported.

\section{Tests of physical performance}

We used a range of tests to measure physical performance of the lower extremities. The SPPB includes strength (five chair stands), endurance (4 m gait speed) and balance (side by side, semitandem and tandem).
Each component is scored from 0 to 4 and is summed SPPB scores between 0 (poor) and 12 (best) performance. ${ }^{23}$ The $6 \mathrm{MWT}$ was used as a measure of aerobic capacity (distance walked reported in meters) and was performed according to recommendations from the American Thoracic Society. ${ }^{24}$ To avoid a ceiling effect and to test muscle endurance, the number of complete getting up and sitting down repetitions performed in $30 \mathrm{~s}$ (30 s sit-to-stand (STS $30 \mathrm{~s}$ )) was also tested. ${ }^{25}$ Muscle strength was measured with the one repetition maximum (1-RM) test using a bilateral leg extension machine for the quadriceps. ${ }^{26}$ 
Adverse events

AEs were defined a priori and categorised as serious (death, cardiac event, hospitalisation, disability or any life-threatening event) or other (musculoskeletal injury, hypoglycaemia, hypotension, hypertensive urgency (>200 $\mathrm{mm} \mathrm{Hg}$ systolic or $110 \mathrm{~mm} \mathrm{Hg}$ diastolic), loss of consciousness, dialysis access complications or any intervention by HD unit staff beyond minimal ultrafiltration). The primary analysis of AEs compared the frequency of events during the exercise session by randomisation group. In a sensitivity analysis, all events occurring during the 12-week intervention period was planned. In both analyses, only the first event per individual was counted (for each type of AE).

\section{Statistical analysis}

We summarised baseline data using percentages, medians and IQR, or mean \pm SD. For secondary outcomes, we explored the effect of aerobic and resistance exercise on QoL and tests of physical performance using the absolute change in score at 12 weeks relative to baseline. To attain the efficiency of the factorial design, all participants who received the aerobic intervention (cycling and the combined group) were compared with all those who did not (resistance and control exercise group) and a similar approach was used for the resistance training group. ${ }^{27}$ Analysis of covariance (ANCOVA) was used to adjust for the baseline score and the other intervention (main effect term) ${ }^{28}$ To correct for multiple comparisons in the combined exercise group, the Bonferroni procedure $(\mathrm{p}<0.025)$ was used. We also estimated the CI for the interaction term for the main study's primary outcomes. ${ }^{27}$ Analyses comparing the groups at follow-up were conducted on an intention to treat basis. Missing outcome data were imputed using a last value carried forward approach. Data analyses were performed using Stata Statistical Software, V.13 MP software (http://www.stata.com).

\section{RESULTS}

This trial is reported according to the Consolidated Standards of Reporting Trials (CONSORT) guidelines ${ }^{29}$ and the recommendations for good practice for the design and analysis of pilot studies. ${ }^{30}$

\section{Participant flow}

Of the 100 patients screened for eligibility, 36 did not meet inclusion criteria and 33 declined to participate (figure 1). The most common reason for exclusion was inability to provide consent $(n=8)$ and the most common reason for declining participation was "no interest in exercising during dialysis' $(n=11)$. A total of 31 participants were randomised and 26 completed the study (cycling, $\mathrm{n}=7$; resistance training, $\mathrm{n}=6$; combined cycling and resistance training, $\mathrm{n}=7$; stretching $\mathrm{n}=6$ ) . Complete outcome data were available for 27 participants.
Baseline characteristics for RCT participants are shown in table 2. Participants were predominantly male $(77 \%)$, Caucasian $(61 \%)$, with a median age of 57.6 years (IQR 49.2-75.1). The primary cause of ESRD was glomerulonephritis $(32.3 \%)$ followed by diabetes (22.6\%). In total, $48 \%$ of participants were diabetic, $90 \%$ had hypertension, $26 \%$ had coronary artery disease and $45 \%$ of trial participants were taking a $\beta$-blocker. Overall, baseline physical functioning was low (mean PCS score of $35 \pm 8$ ) and $39 \%$ of trial participants reported that they never exercised during their leisure time. Out of 31 RCT participants, 25 participated in interviews (2 declined, 1 had a language barrier and 3 changed location or dialysis modality).

The median age of patient interview participants was 57.5 years (IQR, (IQR) 49.2-68.0); participants were primarily male $(76 \%)$ and Caucasian $(64 \%)$. Seven dialysis unit staff participated in pretrial interviews (2 licensed practical nurses (LPNs), 2 registered nurses (RNs), 2 service workers and 1 technician); $86 \%$ were female. During the trial, 11 dialysis unit staff were interviewed (2 LPNs, 8 RNs and 1 technician); 91\% were female. Two dialysis unit staff participated in both sets of interviews.

\section{Feasibility}

Feasibility outcomes are shown in table 1. To highlight key themes regarding the trial's feasibility, exemplar quotes from the interviews of staff members and patients are shown in boxes $1-4$.

\section{Dialysis unit staff (pretrial interviews): barriers to implementation and in servicing}

Although none of the staff members who were interviewed had received any prior formal education on IDE, most staff were not interested in attending an educational session. The preferred means of obtaining more information on IDE were by reviewing 'scientific data' in their own time. Several staff preferred a practical approach to in servicing and suggested that we focus on teaching them how to set up the exercise equipment and complete study documentation (box 1).

All staff members described potential benefits of IDE, such as improved dialysis and leg cramps, weight loss, increased confidence and patients 'keeping busy'. However, it was common for staff to express concern that for many patients in the unit, IDE would be unsafe or would interfere with aspects of the dialysis treatment (box 1). Several staff also expressed concern that the exercise equipment would have a negative impact on their workspace.

\section{Dialysis unit staff (pretrial interviews): selection of suitable candidates}

Several staff emphasised the importance of selecting appropriate patients for IDE, typically referring to those patients who were stable during HD or younger. Several staff members requested that prior to enrolling a patient, we discuss the patient's suitability for the trial with them (box 1). 


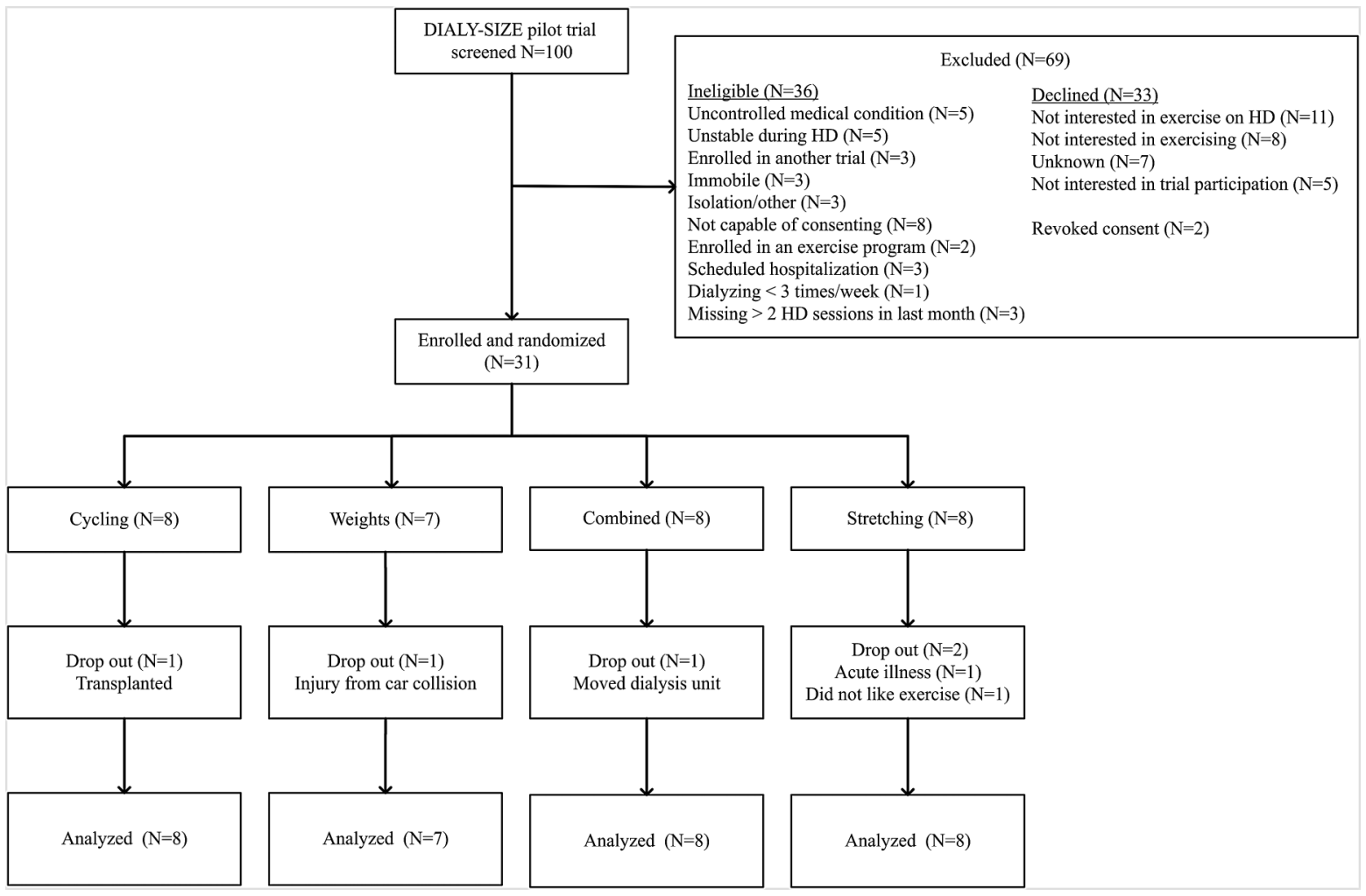

Figure 1 RCT participant flow.

Table 2 Baseline characteristics of trial participants

\begin{tabular}{llllll}
\hline & All $(\mathbf{n = 3 1})$ & Cycling $(\mathbf{n}=\mathbf{8})$ & Weights $(\mathbf{n}=\mathbf{7})$ & Combined $(\mathbf{n}=\mathbf{8})$ & Stretching $(\mathbf{n}=\mathbf{8})$ \\
\hline Age $^{*}$ & $57.6(49.2-75.1)$ & $66.9(55.8-82.4)$ & $59.7(45.9-81.4)$ & $60.3(54.7-68.4)$ & $49.3(43.0-62.3)$ \\
Sex (male) & $24(77)$ & $8(100)$ & $6(86)$ & $3(38)$ & $7(88)$ \\
Time on HD (years) & $3.2(1.7-4.4)$ & $3.7(2.4-4.6)$ & $2.8(2.0-4.0)$ & $2.9(0.7-2.3)$ & $3.3(1.2-6.2)$ \\
Ethnicity & & & & \\
$\quad$ Caucasian & $19(61)$ & $7(88)$ & $3(43)$ & $5(63)$ & $4(50)$ \\
Southeast Asian & $4(13)$ & $1(13)$ & $1(14)$ & $1(13)$ & $2(25)$ \\
Aboriginal & $3(10)$ & 0 & $2(29)$ & 0 & $1(13)$ \\
Other & $5(16)$ & 0 & $1(14)$ & $2(25)$ & $1(13)$ \\
Cause of ESRD & & & & & \\
$\quad$ Diabetes & $7(22.6)$ & $2(25)$ & $1(14.3)$ & $2(25)$ & $2(25)$ \\
Glomerulonephritis & $10(32.3)$ & $1(12.5)$ & $5(71.4)$ & $4(50)$ & 0 \\
Hypertension & $1(3.2)$ & $1(12.5)$ & 0 & 0 & 0 \\
Polycystic kidney disease & $3(9.7)$ & $1(12.5)$ & 0 & $1(12.5)$ & $1(12.5)$ \\
$\quad$ Reflux/urological & $3(9.7)$ & $1(12.5)$ & 0 & 0 & $2(25)$ \\
$\quad$ Other & $5(16.1)$ & $2(25)$ & $1(14.3)$ & 0 & $2(25)$ \\
$\quad$ Unknown & $2(6.5)$ & 0 & 0 & $1(12.5)$ & $1(12.5)$ \\
BMI & $24.7(21.6-29.9)$ & $23.6(22.2-25.7)$ & $25.9(24.6-29.9)$ & $25.3(20.0-30.8)$ & $24.2(20.4-33.8)$ \\
Diabetes & $15(48)$ & $3(38)$ & $3(43)$ & $5(63)$ & $4(50)$ \\
Hypertension & $28(90)$ & $8(100)$ & $7(100)$ & $7(88)$ & $6(75)$ \\
$\beta$ blocker & $14(45)$ & $4(50)$ & $4(57)$ & $3(38)$ & $3(38)$ \\
Coronary artery disease & $8(26)$ & $4(50)$ & $1(14)$ & $2(25)$ & $1(13)$ \\
Heart failure & $7(23)$ & $4(50)$ & $3(43)$ & 0 & 0 \\
QoL-PCS & $35 \pm 8$ & $35 \pm 9$ & $32 \pm 9$ & $35 \pm 10$ & $36 \pm 3$ \\
Never exercise in leisure time & $12(39)$ & $3(38)$ & $4(57)$ & $1(13)$ & $4(50)$ \\
\hline
\end{tabular}

*Median (IQR interval); $\mathrm{N}$ with (\%) or mean ( $\left.{ }^{*} \mathrm{SD}\right)$; totals do not always add to 100 due to rounding.

$\mathrm{HD}$, haemodialysis; BMI, body mass index; ESRD, end-stage renal disease; QoL-PCS, quality of life-physical component summary. 
Box 1 Quotes from pretrial interviews with dialysis unit staff

Barriers to implementation and in servicing

In-servicing

'I would prefer to read, it's easier. And to have it always in my pocket--a reference'.

'As long as we know what-where the documentation's required; I don't think anything else to be honest with you'

Patient safety and staff workflow

'The other thing you're going to have is once the patients start moving about, if they've got their fistula access, it is going to be compromised, and I would not compromise that.

'They could slip out of their chair; they're not sitting properly, they could split their shin with it because they're diabetic, that could cause problems for them...'.

'And we are very, very busy, [at changeover] and nothing's supposed to be around us because we're running from machine to machine to get ready for the next patient.

Selection of suitable candidates

'Some young people [would be good for IDE]... But I cannot say how many.

"I think just being very careful who you pick for the study. It has to be somebody who's physically able to do it, mentally competent. Some people might seem like they're physically able, but they're not mentally able'.

'Actually, [pause] just asking for input on patients to make sure that they are suitable... Or even before you ask them, make sure they are suitable for that program [IDE]. Because I mean, there's a lot of patients they aren't stable and their blood pressure will drop...'

Patients' decision to participate in IDE

'So I think because a lot of them are friends here, so they talk, and, you know, if you're doing that, "What do you think about it?" So they ask each other. Or they can even do it together if they're sitting side-by-side; you know, "Oh, that's kind of fun."... 'Cause a lot of things happen that way here, 'cause they listen to what other patients talk to nurses about, then they think, "Oh, okay, l'll try that, too"'.

'After the conversation with research person, they usually ask our opinion'.

\section{Patients' decision to participate in IDE}

Several staff stated that patients' social networks in the unit were an effective means of disseminating information. Another staff member stated that after being approached for study participation, patients commonly elicited their opinion (box 1).

Based on the data from the pretrial interviews, modifications were made to the study protocol (table 4).

\section{RCT participants: recruitment}

We exceeded the target accrual of 28 participants over 12 weeks. Randomisation to exercise intervention was not a barrier to participation. Patient interview participants reported that recruitment posters displayed outside of the unit and hearing other participants discuss their participation in the trial were effective means of promoting interest and participation in the study (box 2).
Box 2 Exemplar quotes from RCT participants on trial recruitment

Patients' decision to participate in IDE

'No, hadn't thought about-well, I saw the posters and thought, "Hm, interesting. Maybe... I hadn't figured you could do anything... [on dialysis].

'First of all, it was a novelty, and then it was interesting to see how it was a wave of interest; it was a domino effect. And there was a real nice buzz...The [other] patients were, "Hey, you're doing — what are you doing?" etc., etc., so that was super'.

Box 3 Exemplar quotes from interview participants on the impact of the intervention

Dialysis unit staff (midtrial interviews)

The benefits of intradialytic exercise (IDE)

'A lot of them-well, I think probably all of them increased their muscle mass and they have more strength at the end of the program, so they were quite pleased.

'So yeah, the patients, I find, like the ones on the study feel good about themselves. They feel good, and I think they feel better...'

Randomised control trial (RCT) participants

Acceptability of the exercises

'I thought it was-everything was set up perfectly for me. I could do each exercise. Of course, it's a little cumbersome doing a few of the leg reps in a chair, but it's not insurmountable, by far.

'Well, because all I had to do was the stretches, in a way, it was kind of boring, I think. But it's not like stretches aren't good for you; I mean, it is, they're good for you. But I don't know, it's just -it was alright; I wouldn't say it was all that exciting or anything'.

'...I was quite amazed that even with the stretchy bands-and it's a good thing I started with those to kind of loosen me up a little, because I was-like, I had muscles that were sore...'

The benefits of IDE

'I'm more steady on my feet. My legs were pretty shaky before, and now they're not.

'..even my wife has noticed l've got more muscle tone on my legs. And I was really surprised about that, 'cause I didn't think dialysis patients could-and especially even at my age get that kind of deal. But I even noticed myself, I do have more muscle tone'.

'...Like, I do a fair amount of walking, myself, probably 12 blocks a day, and so my legs were fairly good, but I cannot keep up to my wife if we went shopping. Now I can'.

'Oh, I get cramps. Every dialysis run, I had cramps, but after doing exercise, I-no more cramps now'.

'I had restless leg, and I still have it, but surprisingly, not as drastic...'

\section{Dialysis unit staff (mid-trial interviews): fidelity to the} protocol

Although the physical demand of delivering the exercise equipment to patients was not described as onerous, data collection for the trial was. One staff stated that there were occasions when trial documentation 'didn't get done'. Several staff reported that there 
Table 3 Exercise parameters for the four exercise groups

\begin{tabular}{lllll}
\hline & Cycling & Weights & Combined & Stretching/control \\
\hline Borg (Intensity, RPE) & $13 \pm 1$ & $13 \pm 1$ & $13 \pm 1$ & $8 \pm 2$ \\
Mean amount of exercise performed & $28.0 \pm 3.4 \mathrm{~min}$ & $36 \pm 12$ (repetitions) & $27.5 \pm 8.8 \mathrm{~min}$; & NAP \\
& & $5.0 \pm 3.4$ (lbs) & $35 \pm 12$ (repetitions) & \\
Systolic BP $(\mathrm{mm} \mathrm{Hg})$ & & & $3.7 \pm 1.8$ (lbs) & \\
& Pre: $136 \pm 20$ & Pre: $123 \pm 26$ & Pre: $121 \pm 28$ & Pre: $119 \pm 22$ \\
& During: $150 \pm 26$ & During: $127 \pm 27$ & During: $126 \pm 24$ & During: $119 \pm 22$ \\
& Post: $130 \pm 21$ & Post: $117 \pm 26$ & Post: $116 \pm 26$ & Post $118 \pm 20$ \\
Diastolic BP $(\mathrm{mm} \mathrm{Hg})$ & Pre: $74 \pm 16$ & Pre: $66 \pm 15$ & Pre: $62 \pm 13$ & Pre: $70 \pm 14$ \\
& During: $80 \pm 19$ & During: $67 \pm 16$ & During: $67 \pm 13$ & During $70 \pm 15$ \\
& Post: $75 \pm 16$ & Post: $63 \pm 15$ & Post: $63 \pm 13$ & Post $69 \pm 14$ \\
Heart rate $(\mathrm{bpm})$ & Pre: $66 \pm 14$ & Pre: $71 \pm 12$ & Pre: $69 \pm 11$ & Pre: $78 \pm 17$ \\
& During: $85 \pm 20$ & During: $78 \pm 13$ & During: $79 \pm 13$ & During: $77 \pm 16$ \\
& Post $77 \pm 17$ & Post: $74 \pm 13$ & Post: $73 \pm 11$ & Post: $77 \pm 17$ \\
\hline
\end{tabular}

$\mathrm{BP}$, blood pressure; lbs, pounds; RPE, rating of perceived exertion.

Pre, post, and during exercise BP and HRs are a means \pm SD for initiated exercise sessions.

were technical challenges with retrieving $\mathrm{HR}$ and $\mathrm{BP}$ data for DCFs from the HD machines. Some staff also mentioned that recording the vital signs was too time-consuming.

Unit staff frequently made reference to the study as 'just one more thing' and trial resource material was not frequently accessed. Although some staff members felt prepared to assist with the trial, several staff suggested that a lack of clarity on trial processes was a barrier to their involvement (box 4).

\section{RCT participants: fidelity to the protocol}

The dropout rate over the study period was lower than our prespecified threshold at $16 \%$. Irrespective of exercise group allocation, patients' willingness to participate in IDE and their adherence to the exercise prescription was high; of the 1038 training sessions offered, $87 \%$ of sessions were initiated $(89 \%$ in the cycling group, $83 \%$ in the weights group, $90 \%$ in the combined group and $86 \%$ in the stretching group). The exercises were performed as per protocol within all four groups for $>80 \%$ of exercise sessions (table 1). Exercise parameters are shown in table 3 . For the active intervention groups, the mean RPE was within the targeted range and $\mathrm{BP}$ and HR followed a similar trend: increasing during exercise and returning towards baseline post exercise. For the attention control, HR and BP were unchanged over the exercise period.

Although the exercises were protocolised, many participants viewed the intervention as tailored to their level

Table 4 Modifications to the study protocol following pretrial interviews with unit staff

\begin{tabular}{|c|c|c|}
\hline $\begin{array}{l}\text { Trial protocol } \\
\text { item }\end{array}$ & Initial plan/barrier & Modification \\
\hline In-servicing format & $\begin{array}{l}\text { Didactic sessions on the benefits of exercise in } \\
\text { people with ESRD and one practical session } \\
\text { with the exercise equipment }\end{array}$ & $\begin{array}{l}\text { Two practical in-services on study procedures and } \\
\text { equipment set-up } \\
\text { Video posted on YouTube on the exercises and how } \\
\text { to assist patients with equipment set-up } \\
\text { Education materials (articles, pamphlets, summaries) } \\
\text { on IDE placed on the unit for staff }\end{array}$ \\
\hline $\begin{array}{l}\text { Workspace safety } \\
\text { for staff }\end{array}$ & Exercise equipment as workspace hazard & $\begin{array}{l}\text { Unit staff identified where equipment would be stored } \\
\text { on the unit } \\
\text { With unit staff input, protocols for equipment set-up } \\
\text { and removal were written into study protocol }\end{array}$ \\
\hline Recruitment & Only study staff selects suitable candidates & $\begin{array}{l}\text { Prior to enrolling a patient, the charge nurse was } \\
\text { consulted regarding any dialysis-related safety } \\
\text { concerns }\end{array}$ \\
\hline Implementation & $\begin{array}{l}\text { Include several unit staff members as volunteer } \\
\text { 'exercise champions' to lead unit staff and liaise } \\
\text { with study staff }\end{array}$ & $\begin{array}{l}\text { No volunteers found. Identified four staff 'point } \\
\text { people' who were already in leadership roles in the } \\
\text { unit to informally check in with study staff on trial } \\
\text { implementation }\end{array}$ \\
\hline
\end{tabular}

ESRD, end-stage renal disease; IDE, intradialytic exercise. 
Box 4 Exemplar quotes from interview participants on fidelity to the protocol

\section{Dialysis unit staff (midtrial interviews)}

'...we check patients every half-hour for their blood pressures and all the dialysis machine readings and stuff like that, so I find also recording the blood pressure is very time-consuming, because we can go back and look at the list of blood pressures on their machine after, but then we just go back and find them or you have to be recording them every 5 or so minutes, so you're running back and forth between doing your other work and so forth. So I find it's very busy in that respect.

'It was just difficult to add something for us to do, 'cause initially, I think what the thought was to teach all the nurses what the patients were supposed to be doing, but it was just difficult to in-service everybody. They were, like, "Okay, so this is how you fill out the sheet"- cause the sheet, to me, l'm so confused working with it. And sometimes-oftentimes, we're short-staffed, so we don't have the staffing to even get this equipment and all that kind of stuff. So it ended up being they just ended up coming every run and doing the exercise study with the patients.

I think there was a lot of resistance from staff to really help out with it.

'I am prepared because they also have an in-service, and they also have [the kinesiologist] here to show us, she also give us e-mail and show with the video, show us how the exercise going. But I be honest, we don't have time to look at that. We don't have time to sit down and look at that video'!

RCT participants (post-trial participation)

'Yes, because I was starting from zero exercise, so I wasn't sure how much, how hard it would get, how-if I could keep up to what they wanted, that kind of thing...But they did it very gradual, and [the kinesiologist] was very good about telling us ahead of time when they're going to put up the weights or when they're going to increase the minutes of pedaling, so you knew what to expect.

'Well, we were increased at our own pace, which I really liked, because I just went at my own level.

'Also I want to tell you that I have a treadmill at home, but sometimes I do it, sometimes I don't. But here, it's, like, we have to...'

(box 4). Individualised instruction, progression based on RPE and support from the kinesiologist ${ }^{21}$ were commonly mentioned as strengths to the exercise programme. For several patients, knowing there was the expectation of having to exercise facilitated adherence (box 4).

Of the 1038 exercise sessions that were offered, only three were terminated early. In all exercise groups, the most common reason for not initiating a given session was a physical symptom ( $7.5 \%$ of all prescribed sessions), commonly fatigue or feeling generally unwell. HD-related issues accounted for only $1 \%$ of non-initiated sessions, primarily due to central venous catheter dysfunction. Many patients mentioned that consistently obtaining exercise equipment from unit staff was the main barrier to exercise participation; ${ }^{21}$ however, this reason for non-participation was not captured with the exercise DCFs. Only 1.5\% of DCFs had missing data for reason not initiated.
Dialysis unit staff (mid-trial interviews): impact of the intervention

Overall, dialysis unit staff agreed that the exercise programme was valuable for patients (box 3). Their perception of benefit was based on patient report, as the trial results were not known at the time of their interviews. Staff viewed patients' subjective improvements, such as 'feeling healthier', as valid evidence of the benefits of IDE (box 3).

\section{RCT participants: impact of the intervention}

Across all exercise groups, the patients' response to exercise was highly favourable (box 3); $92 \%$ of participants reported they wanted to continue IDE after the trial and $63 \%$ wanted to continue exercising with their current regimen (table 1). There were no crossovers during the trial and no change in the amount of physical activity performed outside of HD time was detected. Concealment of stretching as an active treatment was successful among patients and staff. One participant in the attention control withdrew from the study because he did not find stretching beneficial, 'it wasn't straining, it was just too easy'. Although another participant stated that stretching was 'boring', most participants in the control group viewed stretching as an important aspect of an exercise regimen (box 3). One participant commented that their exercise routine was shorter than the other groups resulting in relatively less interaction time with the kinesiologist.

Patients commonly discussed the benefits of IDE, and for many, these results motivated them to continue exercising (box 3). Patients discussed the exercise-related benefits of IDE, such as greater strength and endurance. Several patients attributed improvements in daily functioning to participation in IDE. Improvements in dialysisrelated symptoms were also mentioned, primarily decreased cramping and restless legs. The most frequently discussed benefit of IDE was that it 'helped kill the four hours' and that it made the time on dialysis more enjoyable. For one participant, IDE served as 'an escape from the humdrum?

\section{Secondary outcomes}

The absolute differences in scores for secondary outcomes are shown in table 5. Scores are presented as crude mean differences and main effects. No significant differences from baseline to 12 weeks were found in the PCS or MCS components of the SF-36 or physical performance tests (6MWT, STS $30 \mathrm{~s}, 1-\mathrm{RM})$. For the main effects analysis of the SPPB, the absolute difference in score and $(95 \% \mathrm{CI})$ was $1.7(0.2$ to 3.3$)$ for the of all those allocated to receive cycling (cycling plus both interventions) versus no cycling (weights plus the attention control) and 1.6 (0.05 to 3.2) for the main effect of those allocated to receive weights versus no weights. This result is consistent with a minimal clinically important difference (values from 0.5 to 1.3 have been recommended). ${ }^{23} 31$ Interaction terms for the planned 
Table 5 Secondary outcomes (QoL, tests of physical performance and strength)

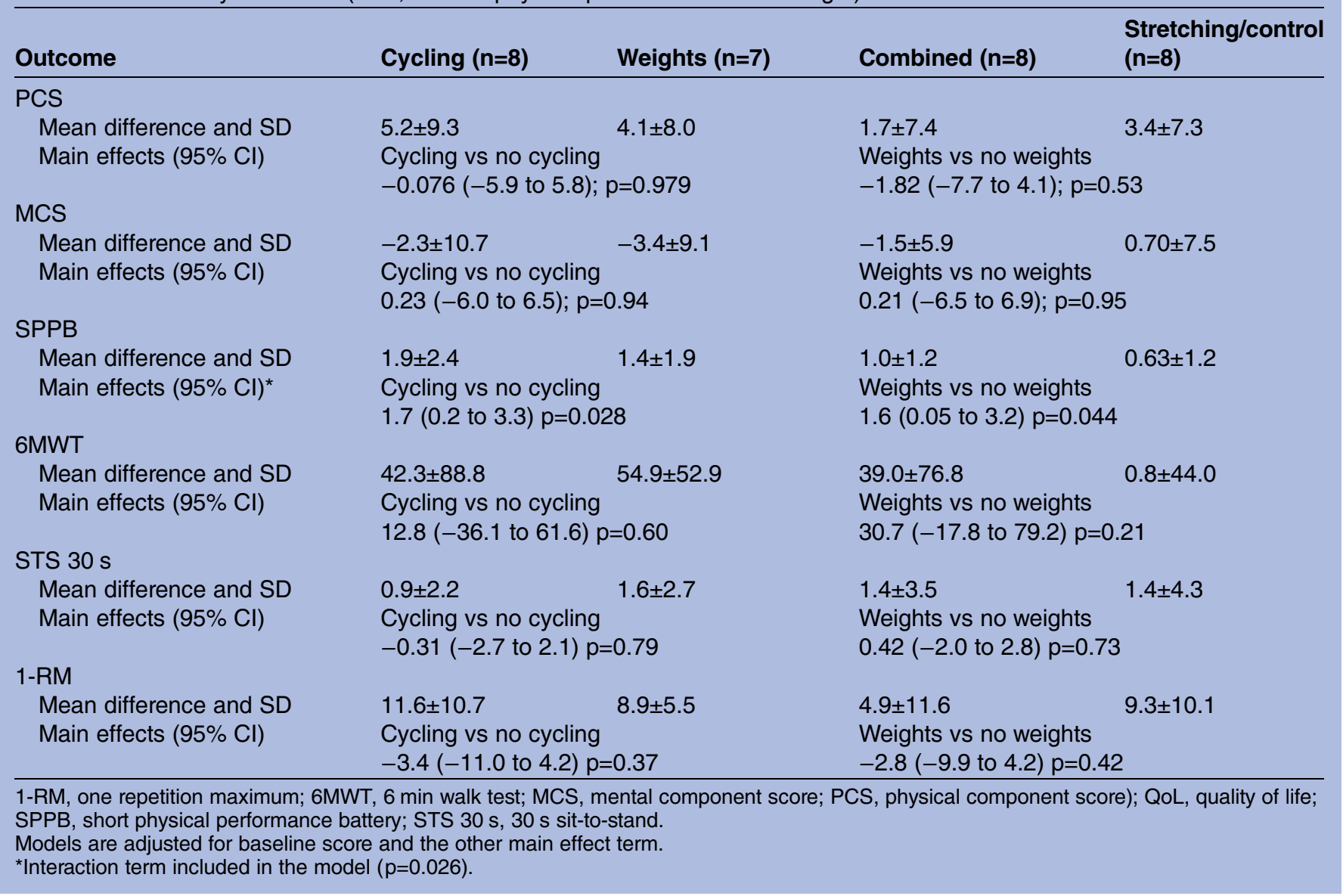

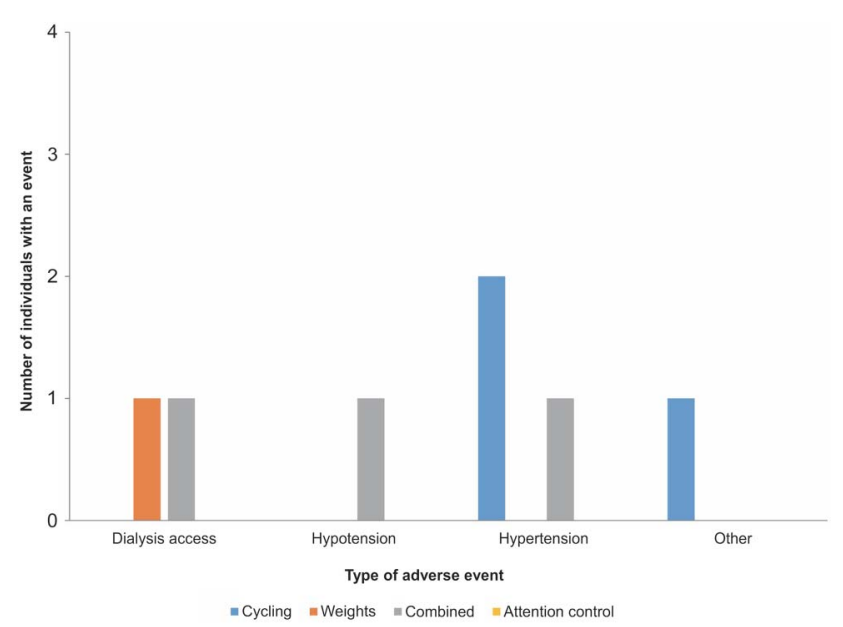

Figure 2 Adverse events occurring during the exercise session. Dialysis access complications=reneedling, haematoma.

primary outcomes of interest for the main study were as follows: PCS -4.2 (-16.1 to 7.6$) ; \mathrm{p}=0.47$ and SPPB -2.9 $(-5.5$ to -0.38$) ; \mathrm{p}=0.026$.

No serious AEs were reported during the exercise sessions. Owing to the low frequency of events in the trial overall, comparative statistics were not performed. AEs occurring during exercise are shown in figure 2. Two patients in the combined group had AEs (one dialysis access complication, one episode of hypertensive urgency and one episode of hypotension). Two patients in the cycling group had AEs (two episodes of hypertension and ankle abrasions from the bike). In the weights group, there was one episode of access complication. There were no AEs during exercise in the stretching group. The overall frequency of AEs was low (figure 3). Notably, there were two episodes of hypotension in the control group, three in the cycling group and one episode in the weights and combined exercise group.

\section{DISCUSSION}

The purpose of this pilot study was to evaluate the feasibility of an IDE exercise intervention and to perform an exploratory analysis of cycling and weight training each compared with control on QoL, tests of physical performance and strength. We demonstrated feasibility of recruitment and high patient acceptability. In addition, few exercise trials in this population have attempted to blind participants to group allocation. ${ }^{4}$ We demonstrated a low risk of contamination and attrition with the use of an attention control and blinding to study hypothesis. However, primarily based on the findings from the interviews with dialysis unit staff and trial participants, several modifications to the study protocol are required prior to proceeding with the main study. 


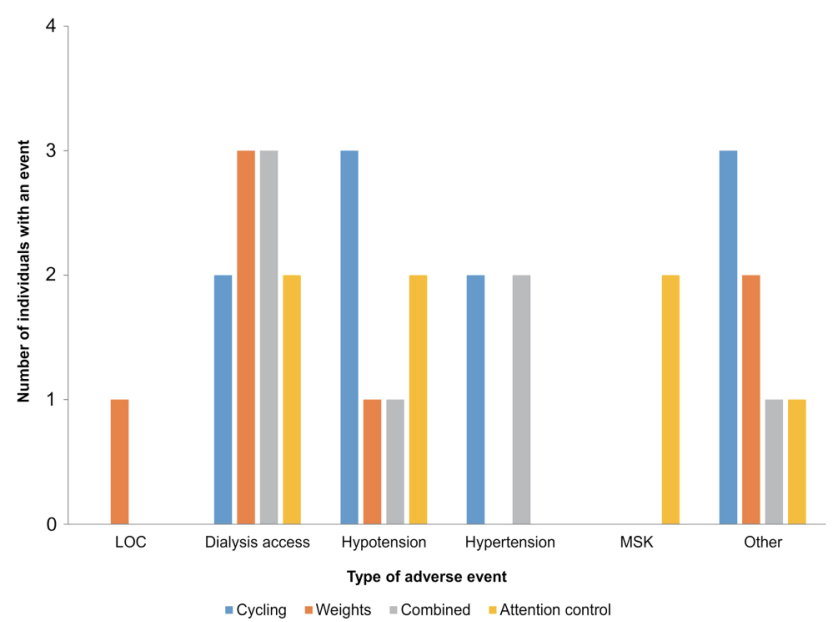

Figure 3 Adverse events occurring over the study period. Dialysis access complications=reneedling, haematoma.

Readiness for change is considered critical to the successful implementation of complex interventions in healthcare settings. ${ }^{32}$ In this pilot, we found that there was a lack of readiness among dialysis unit staff for IDE. Several of the factors that influenced unit staff's preparation, motivation and ability to participate in this trial have been cited in other studies as barriers to the implementation of clinical IDE programmes: lack of time, ${ }^{33} 34$ high patient care demands ${ }^{35}$ and safety concerns with the exercise equipment in their workspace. ${ }^{33}$ In our previous study, we also identified a lack of support from management and personal beliefs about exercise as influencing staff readiness for IDE. ${ }^{21}$ Therefore, prior to recruitment for the main study, it will be necessary to develop a strategy for understanding staff readiness at potential study sites. Although the influence of education on staff participation in IDE remains unknown, in one study, patient and staff thought that a better understanding of IDE would have improved their initial participation. ${ }^{35}$ In this pilot, the lack of interest among many unit staff for IDE education was a barrier to engaging staff. Other more convenient forms of delivering education, that is, videos online and reading material on the unit were not highly accessed. As unit staff expressed that seeing and hearing the benefits from their patients first-hand positively influenced their perceptions of the intervention, a pretrial demonstration phase may be the most effective means of promoting acceptability of IDE. Despite the concerns expressed in the pretrial interviews about patient and workspace safety, that no unit staff mentioned these concerns in the second set of interviews (once the intervention was established), also supports the value of providing staff with the opportunity to experience IDE in their own setting prior to study start.

In addition to requiring the unit staff's assistance with IDE delivery for practical reasons, we identified other reasons why their participation was important. First, due to their frequent and prolonged contact with patients, dialysis unit staff are in a unique position to assist patients with decision-making. ${ }^{36}$ As we found that some patients seek the opinion of dialysis unit staff on study participation, it is important that those who engage in these discussions are prepared to discuss the risks and benefits of IDE with patients. Although $30 \%$ non-participation is comparable to other trials in this population, ${ }^{16181937}$ it is possible that the staff's perceptions of IDE influenced patients' decision to participate. Second, the patients' perspective that unit staff's assistance and encouragement with IDE is consistent with their role as carer and patient advocate has the potential to influence patient acceptability of IDE. ${ }^{21}$ Third, many patients experienced difficulty consistently obtaining exercise equipment from unit staff, which has clear implications for patient adherence. $^{21}$

For unit staff, exercise data collection was too timeconsuming and resulted in missing data. This issue was recognised early in the trial and resolved with greater involvement from study staff. This strategy is not feasible for a multisite study and exercise vital signs will be limited to pre-exercise, midexercise and postexercise. We also found that for unit staff, feasibility of workflow integration was affected by the timing of when in the dialysis treatment that IDE was performed. ${ }^{21}$ To decrease the risk of hypotension, other trials have typically completed exercise within the first $1-2$ hours of the HD session $^{16} 38{ }^{39}$ and starting exercise within the first hour of HD is often recommended. However, this is often the busiest time for unit staff, and in settings where there are staffing constraints, it may be a barrier to optimal staff engagement. We are only aware of one trial where IDE was performed in the final 2 hours of the HD session and this was well tolerated. ${ }^{40}$ Our protocol specified that patients finish their exercise within the first 3 hours of the dialysis shift. The safety of this approach is supported by our BP and safety data. A more detailed evaluation of the timing of the HD session and its effect on BP would provide important insight into how to optimise the safety and the practicality of IDE delivery.

This study has several important strengths. Most studies evaluating exercise adherence in people with kidney disease have focused on individual determinants and not evaluated programme factors. ${ }^{41}{ }^{42}$ In this study, progression based on RPE and individualised instruction facilitated acceptability among patients. As described in our qualitative study, patients perceived the kinesiologist's technical support as conveying a sense of esteem and capability. ${ }^{21}$ This interaction may have served to increase participation, irrespective of group assignment. Additionally, the most commonly mentioned benefit to IDE was that it helped pass the time, suggesting that many patients are interested in participating in interventions where they can use their time on HD more constructively. It also suggests that some of the perceived improvement in well-being could be mediated through engagement in an activity, rather than exercise. These findings underscore the importance of continuing to use a supervised attention control for the main study. 
Our study also has several limitations that warrant mention. Given the potential impact of the interaction with the exercise specialist on IDE acceptability, it will be important to ensure that the interaction time between the attention control group and the kinesiologist is equivalent to that of the intervention groups. Also, the trial study population was small and relatively homogeneous with respect to sex, age and ethnicity, which may limit the generalisability of the findings.

We did not detect differences in physical activity or exercise performed outside of the unit during the trial, nor was the trial powered for this outcome. The antagonistic interaction term for the SPPB will also need to be explored in more detail, as this could be a spurious finding due to multiple outcome testing. The primary aim of this pilot study was to evaluate feasibility and small sample sizes were used. Therefore, the finding that cycling or weights did not improve QoL or other measures of physical performance should not be interpreted as providing evidence for no effect. Based on $80 \%$ power to detect a difference in the primary outcome of PCS of five points ${ }^{43}$ in the main effect of aerobic and the main effect of resistance, 32 participants per arm are required. Allowing for $25 \%$ dropout per arm, the main study will enrol 160 patients. A four-arm parallel design would allow direct comparisons between the interventions; however, the sample size would need to be at least twice as large as that calculated for the main study. Given that recruitment and retention are barriers to performing adequately powered exercise studies in this population, the factorial design is one means of improving efficiency while allowing for indirect comparisons between aerobic and resistance training.

\section{CONCLUSIONS}

To our knowledge, this is the first feasibility study to use qualitative methods to evaluate IDE implementation within an RCT design and to address known limitations to trial design. In addition to informing the design of our future definitive study, these results are useful in the development of future trials and for guiding clinicians with the implementation of their own IDE interventions. The key lesson learnt was that within this protocolised setting, the potential for unit staff readiness to influence aspects of feasibility, such as recruitment and patient adherence was high. Therefore, prior to study start, more time will need to be invested in understanding and enhancing staff readiness. For engaging unit staff, a less didactic approach that is also integrated into their existing workflow may be highly effective.

\footnotetext{
Author affiliations

${ }^{1}$ Division of Nephrology, University of Alberta, Edmonton, Alberta, Canada

${ }^{2}$ Faculty of Nursing, University of Alberta, Edmonton Clinic Health Academy, Edmonton, Alberta, Canada

${ }^{3}$ Kidney Health Research Group, University of Alberta, Edmonton, Alberta, Canada

${ }^{4}$ Northern Alberta Renal Program, University of Alberta Hospital, Edmonton, Alberta, Canada
}

${ }^{5}$ College of Nursing and Health Innovation, University of Texas at Arlington, Arlington, Texas, USA

${ }^{6}$ Division of Nephrology, University of Calgary, Calgary, Alberta, Canada

Twitter Follow Stephanie Thompson at @StephanieTh11

Contributors Authorship followed International Committee of Medical Journal Editors (ICMJE) guidelines. ST was responsible for the inception and design of the project and prepared the manuscript. MH, SK, AM and MT participated in the design of the study and provided methodological input. IG participated in the design of the exercise intervention. AL provided statistical support. All authors read and approved the manuscript.

Funding This study was funded by a grant from the University Hospital Foundation awarded to MT and a Clinical Research Fellowship award from Alberta Innovates-Health Solutions awarded to ST. The funders had no role in the design, collection, analysis, interpretation, writing or submission of the manuscript.

\section{Competing interests None declared}

Ethics approval Health Research Ethics Board, University of Alberta.

Provenance and peer review Not commissioned; externally peer reviewed.

Data sharing statement No additional data are available.

Open Access This is an Open Access article distributed in accordance with the Creative Commons Attribution Non Commercial (CC BY-NC 4.0) license, which permits others to distribute, remix, adapt, build upon this work noncommercially, and license their derivative works on different terms, provided the original work is properly cited and the use is non-commercial. See: http:// creativecommons.org/licenses/by-nc/4.0/

\section{REFERENCES}

1. Fukuhara S, Lopes AA, Bragg-Gresham JL, et al. Health-related quality of life among dialysis patients on three continents: the Dialysis Outcomes and Practice Patterns Study. Kidney Int 2003;64:1903-10.

2. McFarlane PA, Bayoumi AM, Pierratos A, et al. The quality of life and cost utility of home nocturnal and conventional in-center hemodialysis. Kidney Int 2003;64:1004-11.

3. Kurella Tamura M, Covinsky KE, Chertow GM, et al. Functional status of elderly adults before and after initiation of dialysis. $N$ Eng J Med 2009;361:1539-47.

4. Heiwe S, Jacobson SH. Exercise training for adults with chronic kidney disease. Cochrane Database Syst Rev 2011;(10):CD003236.

5. Sheng $\mathrm{K}$, Zhang $\mathrm{P}$, Chen $\mathrm{L}$, et al. Intradialytic exercise in hemodialysis patients: a systematic review and meta-analysis. Am J Nephrol 2014;40:478-90.

6. Warburton DE, Gledhill N, Quinney A. Musculoskeletal fitness and health. Can J Appl Physiol 2001;26:217-37.

7. Warburton DER, Nicol CW, Bredin SSD. Prescribing exercise as preventative therapy. Can Med J 2006;174:961-74.

8. Warburton DE, Glendhill N, Quinney A. The effects of changes in musculoskeletal fitness on health. Can J Appl Physiol 2001;26:161-216.

9. Mandic S, Myers J, Selig SE, et al. Resistance versus aerobic exercise training in chronic heart failure. Curr Heart Fail Rep 2012;9:57-64.

10. Konstantinidou E, Koukouvou G, Kouidi E, et al. Exercise training in patients with end-stage renal disease on hemodialysis: comparison of three rehabilitation programs. J Rehabil Med 2002;34:40-5.

11. Moore GF, Audrey S, Barker M, et al. Process evaluation of complex interventions: Medical Research Council guidance. BMJ 2015;350: h1258

12. McAlister FA, Straus SE, Sackett DL, et al. Analysis and reporting of factorial trials: a systematic review. JAMA 2003;289:2545-53.

13. Thompson WR, Gordon NF, Prescatello LS. ACSM's Guidelines for Exercise Testing and Prescription. 8th Edition. Baltimore: Lippincott Williams and Wilkins. 2010

14. Borg G. Physical work and effort. Oxford: Pergamon Press, 1977.

15. Schulz KF, Grimes DA. Blinding in randomised trials: hiding who got what. Epidemiol ser 2002;359:696-700.

16. Chen JLT, Godfrey S, Ng TT, et al. Effect of intra-dialytic, low-intensity strength training on functional capacity in adult haemodialysis patients a randomized pilot trial. Nephrol Dial Transplant 2010;25:1936-43.

17. DePaul V, Moreland J, Eager T, et al. The effectiveness of aerobic and muscle strength training in patients receiving hemodialysis and 
EPO: a randomized controlled trial. Am J Kidney Dis 2002;40:1219-29.

18. Segura-Orti E, Kouidi E, Lison JF. Effect of resistance exercise during hemodialysis on physical function and quality of life: randomized controlled trial. Clin Nephrol 2009;71:527-37.

19. Kouidi EJ, Grekas DM, Deligiannis AP. Effects of exercise training on noninvasive cardiac measures in patients undergoing long-term hemodialysis: a randomized controlled trial. Am J Kidney Dis 2009;54:511-21.

20. Fix A, Daughton D. Human activity profile professional manual. Odessa: Psychological Assessment Resources Inc, 1988.

21. Thompson S, Tonelli M, Klarenbach S, et al. A qualitative study to explore patient and staff perceptions of intradialytic exercise. Clin $J$ Am Soc Nephrol 2016;11:1024-33.

22. Hays RD, Kallich JD, Mapes DL, et al. Development of the kidney disease quality of life (KDQOL) instrument. Qual Life Res 1994;3:329-38.

23. Guralnik JM, Simonsick EM, Ferrucci L, et al. A short physical performance battery assessing lower extremity function: association with self-reported disability and prediction of mortality and nursing home admission. J Gerontol 1994;49:M85-94.

24. Crapo RO, Casaburi R, Coates AL, et al. ATS statement: guidelines for the six-minute walk test. Am J Respir Crit Care Med 2002;166:111-17.

25. The 30-Second Chair Stand Test. Centers Dis. Control Prev. Natl. Cent. Inj. Prev. Control. http://www.cdc.gov/steadi/pdf/30_second_ chair_stand_test-a.pdf (accessed 22 Jan 2016).

26. Haykowsky $\bar{M}$, Taylor $\mathrm{D}$, Teo $\mathrm{K}$, et al. Left ventricular wall stress during leg-press exercise performed with a brief Valsalva maneuver. Chest 2001;119:150-4.

27. Montgomery AA, Peters TJ, Little P. Design, analysis and presentation of factorial randomised controlled trials. BMC Med Res Methodol 2003;3:26.

28. Vickers AJ, Altman DG. Statistics notes: analysing controlled trials with baseline and follow up measurements. BMJ 2001;323:1123-4.

29. Moher D, Hopewell S, Schulz KF, et al. CONSORT 2010 Explanation and Elaboration: updated guidelines for reporting parallel group randomised trials. J Clin Epidemiol 2010;63:e1-37.

30. Lancaster GA, Dodd S, Williamson PR. Design and analysis of pilot studies: recommendations for good practice. J Eval Clin Pract 2004;10:307-12.
31. Perera S, Mody SH, Woodman RC, et al. Meaningful change and responsiveness in common physical performance measures in older adults. J Am Geriatr Soc 2006;54:743-9.

32. Weiner BJ. A theory of organizational readiness for change. Implement Sci 2009;4:67.

33. Kontos PC, Miller KL, Brooks D, et al. Factors influencing exercise participation by older adults requiring chronic hemodialysis: a qualitative study. Int Urol Nephrol 2007;39:1303-11.

34. Painter P, Carlson L, Carey S, et al. Determinants of exercise encouragement practices in hemodialysis staff. Nephrol Nurs $J$ 2004;31:67-74

35. Young HML, Hudson N, Clarke AL, et al. Patient and staff perceptions of intradialytic exercise before and after implementation: a qualitative study. PLOS ONE 2015;10:e0128995

36. Brown $S$, Bain $P$, Broderick $P$, et al. Original research emotional effort and perceived support in renal nursing : a comparative interview study. J Ren Care 2013;39:246-55.

37. Johansen KL, Painter PL, Sakkas GK, et al. Effects of resistance exercise training and nandrolone decanoate on body composition and muscle function among patients who receive hemodialysis: a randomized, controlled trial. J Am Soc Nephrol 2006;17:2307-14.

38. Painter P, Moore G, Carlson L, et al. Effects of exercise training plus normalization of hematocrit on exercise capacity and health-related quality of life. Am J Kidney Dis 2002;39:257-65

39. Mohseni R, Emami Zeydi A, Ilali E, et al. The effect of intradialytic aerobic exercise on dialysis efficacy in hemodialysis patients: a randomized controlled trial. Oman Med J 2013;28:345-9.

40. Cheema BS, O'Sullivan AJ, Chan M, et al. Progressive resistance training during hemodialysis: rationale and method of a randomized-controlled trial. Hemodial Int 2006;10:303-10.

41. Williams A, Stephens R, McKnight T, et al. Factors affecting adherence of end-stage renal disease patients to an exercise programme. Br J Sports Med 1991;25:90-3.

42. Greenwood SA, Lindup $\mathrm{H}$, Taylor $\mathrm{K}$, et al. Evaluation of a pragmatic exercise rehabilitation programme in chronic kidney disease. Nephrol Dial Transplant 2012;27(Suppl 3):iii126-34.

43. Norman GR, Sloan JA, Wyrwich KW. Point/counterpoint interpretation of changes in health-related quality of life the remarkable universality of half a standard deviation. Med Care 2003;41:582-92. 